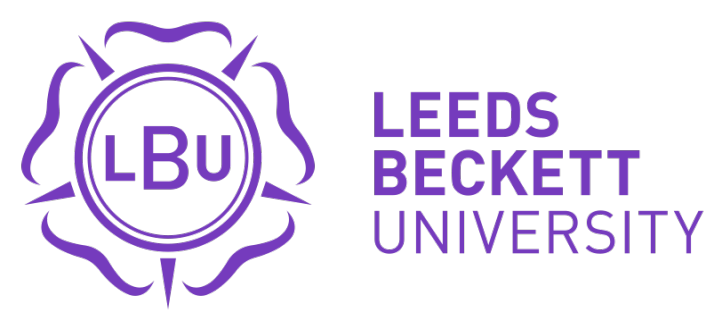

Citation:

Ireson, J and Jones, GL and Winter, M and Radley, S and Hancock, B and Tidy, J (2018) Systematic review of health-related quality of life and patient-reported outcome measures in Gestational Trophoblastic Disease: A parallel synthesis approach. Lancet Oncology. ISSN 1470-2045 DOI: https://doi.org/10.1016/S1470-2045(17)30686-1

Link to Leeds Beckett Repository record:

https://eprints.leedsbeckett.ac.uk/id/eprint/4049/

Document Version:

Article (Accepted Version)

The aim of the Leeds Beckett Repository is to provide open access to our research, as required by funder policies and permitted by publishers and copyright law.

The Leeds Beckett repository holds a wide range of publications, each of which has been checked for copyright and the relevant embargo period has been applied by the Research Services team.

We operate on a standard take-down policy. If you are the author or publisher of an output and you would like it removed from the repository, please contact us and we will investigate on a case-by-case basis.

Each thesis in the repository has been cleared where necessary by the author for third party copyright. If you would like a thesis to be removed from the repository or believe there is an issue with copyright, please contact us on openaccess@leedsbeckett.ac.uk and we will investigate on a case-by-case basis. 


\section{Systematic review of health-related quality of life and patient-reported outcome measures in Gestational Trophoblastic Disease: A parallel synthesis approach}

Corresponding author:

Jane Ireson RN

Sheffield Trophoblastic Centre

Broomcross Building

Weston Park Hospital

Whitham Road, Sheffield

S10 2SJ, United Kingdom

07971907640

Jane.ireson@sth.nhs.uk

Professor Georgina Jones CPsychol

Professor of Health Psychology

Department of Psychology,

School of Social Sciences

Leeds Beckett University

Leeds, LS1 9HE, United Kingdom

Tel: +44 (0)113 8125106

Email: g.l.jones@leedsbeckett.ac.uk

Matthew Winter MD

Consultant Medical Oncologist

Deputy Director of Sheffield Trophoblastic Centre

Weston Park Hospital

Whitham Road, Sheffield

S10 2SJ, United Kingdom

Matthew.Winter@sth.nhs.uk

Tel: +44 (0)1142265183

Stephen Radley MD

Consultant Gynaecologist

Jessop Wing

Sheffield Teaching Hospitals NHS Trust

Stephen.Radley@sth.nhs.uk

Professor Barry Hancock MD

Emeritus Professor

Weston Park Hospital

Whitham Road, Sheffield

S10 2SJ, United Kingdom

b.w.hancock@sheffield.ac.uk

Professor John Tidy MD

Professor of Gynaecological Oncology

Director of Sheffield Trophoblastic Centre

Weston Park Hospital 
Whitham Road, Sheffield

S10 2SJ, United Kingdom

John.tidy@sth.nhs.uk 


\section{Summary}

Gestational Trophoblastic Disease (GTD) is a rare complication of pregnancy that can develop into cancer. Medical outcomes are well researched but evidence is required on the impact of GTD on health-related quality of life (HRQoL) to improve care. The review was conducted to determine the impact of GTD and its treatment on HRQoL and identify how HRQoL is measured and appropriateness of these measures.

Quantitative studies found HRQoL in long-term survivors to be at or above population norms. GTD appeared to have a negative impact on HRQoL where patients experience physical, psychological and social sequelae related to the condition. Clinically significant levels of anxiety, depression, sexual dysfunction and fertility-related distress were found. The results should be treated with caution because the evidence base was limited to small heterogeneous samples, retrospective data and the wide range of measures used. Within the qualitative data, new themes emerged including nerve damage, fatigue, amenorrhea, and grief. Currently, these areas are not captured in patient reported outcome measures (PROMs) and the content may not be valid for this population. Further qualitative research could lead to development of a GTD specific PROM providing reliable, meaningful and valid assessments and allowing longitudinal data to be obtained. 


\section{Background}

Gestational Trophoblastic Disease (GTD) is a rare condition that affects women during or after pregnancy. GTD is an umbrella term for a range of conditions, ranging from the pre-malignant disorders of complete molar (CM) or partial molar (PM) pregnancy, through to the malignant conditions commonly known as Gestational Trophoblastic Neoplasia (GTN), including invasive mole, choriocarcinoma, and the rare placental-site and epithelioid trophoblastic tumour (PSTT and ETT). 1,2

All cases in the UK are registered for human chorionic gonadotropin (hCG) monitoring at one of three designated screening centres, and if subsequent treatment is required, patients are treated at one of the two Trophoblastic Disease Centres in Charing Cross Hospital, London, or Weston Park Hospital, Sheffield. The need for treatment is defined by malignant change and is almost always identified by a rising or plateau in serum hCG concentration, occurring in approximately $15 \%$ of CMs and $0.5-1 \%$ of PMs. ${ }^{2}$

The GTD multi-disciplinary team (MDT) reflects a service that has moved away from a purely biomedical model of health towards patient centred care, supporting patients throughout their experience of GTD. ${ }^{3}$ Emotional support is paramount whilst patients are receiving monitoring and treatment, but outcomes are measured only in the medical domain including the assessment of hCG results, clinician assessed toxicity, nights in hospital, and cycles of treatment. The impact of the diagnosis and treatment on the patient's quality of life is subjective and is not objectively or routinely captured at any stage. As a result all chemotherapy decisions are based wholly on historic data on medical outcomes. Without any medical need to assess the impact of treatment, communication and emotional support drop off sharply once active monitoring and treatment finishes.

Health-related quality of life (HRQoL) is a multi-dimensional concept that represents a patient's general perception of the effects of illness and treatment on physical, psychological and social aspects of life. ${ }^{4} \mathrm{HRQOL}$ is a rapidly evolving field of research, and it is now generally accepted that the patients' perspective is paramount in research and clinical practice.5,6,7 HRQoL outcomes can be captured using patient-reported outcome measures (PROMs). ${ }^{7}$ A PRO is a subjective outcome - referring to anything reported by 'asking the patient', or more formally defined as "any report of the status of a patient's health condition that comes directly from the patient, without interpretation of the patient's response by a clinician or anyone else". ${ }^{4}$ A PRO comprises a variety of outcome measures such as emotional functioning (including anxiety and depression), physical functioning, social functioning, pain, fatigue, other symptoms and toxicity.

Within the United Kingdom (UK) National Health Service (NHS) PROMs were introduced into routine health care in 2009, with the intention of extending routine measurement to a range of conditions including cancer. For policy 
makers within the NHS, PROMs are an opportunity to move away from clinical outcome data that has centred on death and other clinical indicators such as infection rates, readmissions, recurrence, and adverse incidents. ${ }^{8}$

Within NHS cancer services there has been a growth in overall PROM data. Whilst this has been mainly within clinical trials aiming to demonstrate the equivalence or benefit of novel cancer treatments, surgical oncology teams now commonly collect PROM data to inform clinical decision making and patient choice, regulate output and productivity, and inform commissioners on performance. ${ }^{8,9}$ Collecting and analysing PROM data for conditions that require lengthy treatments such as chemotherapy and radiotherapy can be challenging, as they involve complex packages of health and social care rather than a single intervention. As a result there is a difficulty in interpreting the resultant trends from PROMs and attributing any change to particular providers or interventions. ${ }^{8}$

PROM data has proved useful in measuring the short and long-term effects of treatment for cancer patients having complex treatment and the HRQoL of survivors. ${ }^{10,11}$ Current recommendations by the National Cancer Survivorship Initiative (NCSI), All Party Parliamentary Group on Cancer (APPGC) and Macmillan Cancer Support advocate the routine use of PROMs from diagnosis onwards, in order to help clinical teams focus on what really matters to patients, especially at the end of active treatment when patients often feel isolated and abandoned. 10,12 Research supports this recommendation, providing strong evidence in cancer settings that well-implemented PROMs improve patientprovider communication and patient satisfaction, in addition to growing evidence that PROMs improve monitoring of treatment response and the detection of unrecognised problems. ${ }^{13}$

\section{Justification for the Current Review}

Medical outcomes for GTD patients are widely researched and generally excellent, though a review of HRQoL in GTD is lacking. The aim of this systematic review is to evaluate all the existing evidence on the impact of GTD on HRQoL and identify gaps in the evidence. Currently there is no justification for using a particular PROM within GTD. This evidence may support the choice of a preexisting PROM in routine clinical practice or justify research in developing a GTD specific PROM to enable a more patient-focused service.

\section{Methods}

The systematic review was undertaken using the Cochrane group's parallel synthesis approach, ${ }^{14}$ whereby qualitative evidence and quantitative evidence are conducted as separate, but linked, reviews. The qualitative synthesis can be used in parallel and juxtaposed alongside the quantitative synthesis in a process of data triangulation to aid in answering the review question. 


\section{Search Strategy and Selection criteria}

A comprehensive, systematic search of the following databases was conducted in July 2016: MEDLINE 1948- present via OVID plus In-process and other nonindexed citations (all available years); Web of Science "All Databases" "All Years"; PsycINFO 1806- present via OVIDSp In-process and other non-indexed citations; Cochrane Database of Systematic Reviews; PROMS bibliography (Oxford University).

A combination of terms related to the population: 'gestational trophoblastic'; 'molar pregnancy'; 'hydatidiform mole'; 'complete mole'; 'partial mole'; 'choriocarcinoma' 'placental site trophoblastic' and the intervention/outcomes: 'quality of life'; 'sex*'; 'anxiety'; 'depression'; 'psychosocial'; 'psychologic*'; 'fertility'; 'side effects'; 'late effects'; 'survivor'; 'symptom*' were employed using MeSH terms, free text searching and Boolean phrase capabilities.

Exclusion Criteria:

All other types of miscarriage not specified in the population criteria, e.g. all triploid pregnancies, ectopic pregnancies.

Studies where the full text is not available in English.

Studies that measure satisfaction with treatment or management strategies.

\section{Results}

The search retrieved 1400 articles with 17 quantitative and 4 qualitative studies included in the review. Study selection flow is shown in a PRISMA diagram (Figure 1). 


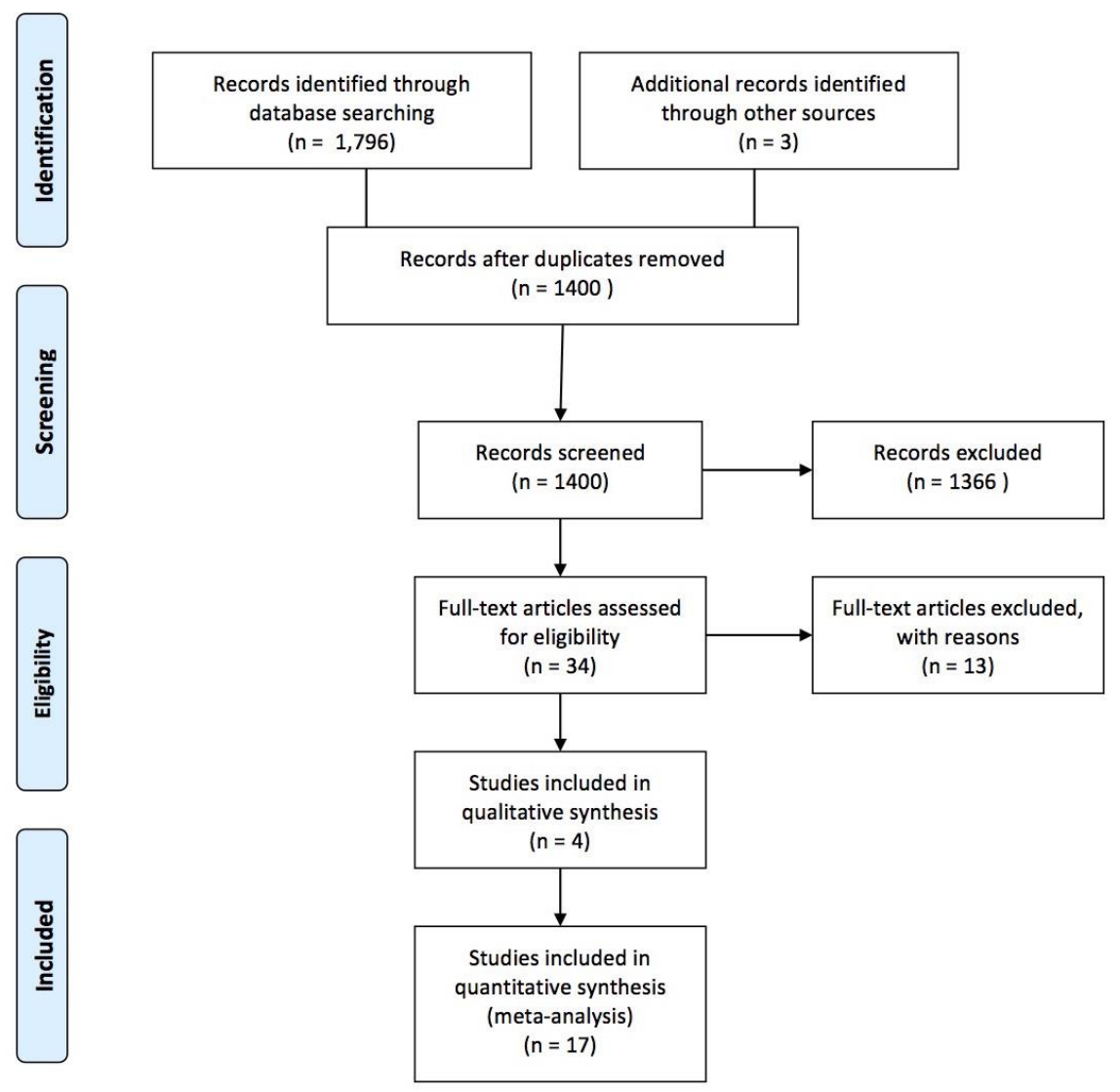

\section{Characteristics of Quantitative studies}

\section{Population}

Of the 17 quantitative studies, eight included a mix of molar pregnancy (MP) and GTN patients, eight included only GTN patients and one included only MP patients. 12 of the 16 studies with GTN patients only included patients who were in complete remission. In the remaining four studies active disease status was not explicit. Women were diagnosed with MP/GTN up to 30 years prior to enrollment. All the studies were cross-sectional. Within the GTN participants $57 \%$ had single agent chemotherapy and 43\% multi-agent chemotherapy.

Figure 2a.

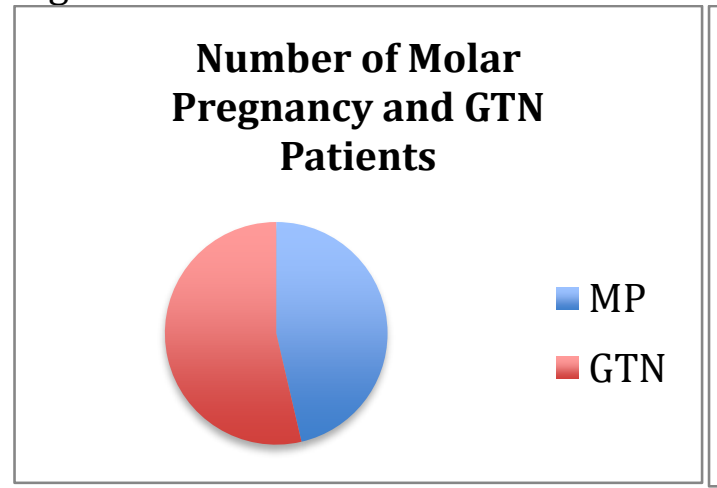

b.

\section{Disease status of GTN/ MP patients}


Thirty-one different instruments were identified. Seven studies developed a study specific questionnaire, or adapted a pre-existing questionnaire. Seven studies used standardised tools including generic to multi-dimensional questionnaires, disease specific questionnaires or instruments for specific aspects of HRQoL such as anxiety, depression, pain or fertility.

See web appendix page 1 for Table I: Summary of PROMs used; and page 3 Table II: Summary of psychometric properties of validated PROMs in GTD

\section{Characteristics of Qualitative Studies}

The qualitative studies included 293 participants, 154 had GTD, 139 had GTN. All studies used an interpretive approach including qualitative data collection and data analysis.

Table III: Summary of qualitative studies

\begin{tabular}{|l|l|l|l|l|}
\hline Study (reference) & Study Setting & Sample size & Sample composition & Data analysis \\
\hline Ngan \& Tang $(1986)^{38}$ & Hong Kong & 105 & GTD \& GTN & NR \\
\hline Flam $(1993)^{37}$ & Sweden & 22 & GTN & Thematic \\
\hline Petersen $(2009)^{15}$ & Australia & 158 & GTD \& GTN & NR \\
\hline Singh et al $(2017)^{36}$ & UK & 8 & GTN & Framework \\
\hline
\end{tabular}

\section{Synthesis of Quantitative Evidence}

\section{Molar Pregnancy (MP)}

This group included women diagnosed with a complete or partial molar pregnancy and undergoing screening at the time of the study, or had been screened in the past, for malignant change.

\section{Quality of Life}

Two studies 15,16 assessed overall quality of life, using a validated generic quality of life measurement tool (SWLS \& WHOQOL-BREF). Although some of the participants may have not been on active follow up, these studies generated results from relatively homogeneous samples of MP patients who perceived their overall quality of life as good.

\section{Physical Functioning}

No study specifically explored the impact on physical functioning of a molar pregnancy. Ferreira et al, using the WHO QOL BREF, 16 found HRQoL domain scores to be lowest for psychologic health 58.33 (95\% CI 41-67-66.67) and highest for physical health 66.82 (SD 16.03) (a higher score indicating better HRQoL on a scale of 1-100). 


\section{Psychological Functioning}

Four studies used standardised measures of anxiety and depression (HADS, BDI, STAI trait/ state, CES-D) and suggest clinically significant levels of anxiety and moderate levels of depression in MP. ${ }^{15-18} 55 \%$ of women in Petersen's study scored $>8$ on the HADS anxiety subscale ( $>8$ signifies presence of clinically significant anxiety/depression ${ }^{19}$ ) only $20 \%$ scored $>8$ on the depression subscale. ${ }^{15}$ The GTD related distress measured was higher amongst the MP group ( $\mathrm{p}=0 \cdot 022) .17$ The non-standardised tools, ${ }^{20,21}$ also demonstrated moderate levels of anxiety, with more than $50 \%$ of MP patients feeling tense about their weekly hCG test. The causes of anxiety and depression were fear of future malignancy, uncertainties about future pregnancy and physical health. ${ }^{22}$ The results suggest similar levels of anxiety and depression across both MP and GTN groups, with evidence that anxiety is clinically significant.

\section{$\underline{\text { Social Functioning }}$}

\section{$\underline{\text { Relationship concerns and sexual function }}$}

Five studies investigated the relationship between a MP patient and their partner, reporting a supportive, and often improved relationship. 15, 17, 20, 21,22 For both MP and GTN patients, there is evidence that not having a pregnancy following the diagnosis increases relationship dissatisfaction $(\mathrm{p}=0 \cdot 01) .{ }^{17}$

Sexual dysfunction was found to affect between $21 \%$ and $53 \%$ of MP patients, although the majority of women did not attribute the dysfunction to the MP. 17,22 Sexual problems were reported as a lack of desire (42\%) and stress (44\%). ${ }^{22}$ The Sexual History Form-12 was used in one study and indicated mild sexual dysfunction, but the reasons for this were not explored. ${ }^{15}$

\section{Fertility concerns}

Women with MP and GTN have non-significant levels of infertility stress when measured by the validated Fertility Problem Inventory (FPI). ${ }^{18}$ However, significant differences in individual items on the score were found between younger and older women and for those with and without children.

The non-standardised measures of fertility concern, which are more specific to GTD, appear to be contradictory. Some show MP patients are not overly worried about infertility, and acknowledge the likelihood of recurrence is minimal, with $92 \%$ wanting to conceive again, ${ }^{21}$ and only $17 \%$ worried about a future pregnancy. ${ }^{20}$ However, Wenzel et al found $50 \cdot 5 \%$ of MP patients fearing becoming pregnant again, and nearly $40 \%$ questioning their ability to have children. ${ }^{22}$

\section{Gestational Trophoblastic Neoplasia (GTN)}

GTN, as a malignant condition, requires further treatment and women are assessed before chemotherapy using the FIGO 2000 scoring system. ${ }^{1}$ 
Chemotherapy can be single or multi-agent and lasts on average for 4-6 months. ${ }^{1}$ A small number of malignant patients undergo surgery as part of their treatment.

The GTN group is more diverse. No single study focuses on a particular FIGO score, type of treatment, or disease burden (metastasis/no metastasis) but gathers together a sample across the GTN spectrum.

\section{General HRQOL}

Seven of the studies, ${ }^{15}, 16,23-27$ that include GTN patients measure HRQoL use a validated generic HRQoL tool (FACT-G, EORTC QLQ-C30, WHOQOL-BREF, SWLS, QoL-CS, SF 36 and the SF 12). Of these, there is a mixture of patients already in remission and patients undergoing active treatment. Where the study only includes patients in remission, ${ }^{24-27}$ the results show levels of HRQoL at or above population norms (Table IV).

Table IV Summary of FACT-G scores from included studies and controls

\begin{tabular}{|l|l|l|l|l|}
\hline & $\begin{array}{l}\text { Wilailak } \\
(2011)^{25} \\
\text { Mean (SD) } \\
\text { Gynaecology } \\
\text { Cancer } \\
\text { survivors }\end{array}$ & $\begin{array}{l}\text { Leenharattanarak } \\
(2014)^{26} \\
\text { Mean (SD) } \\
\text { GTN survivors }\end{array}$ & $\begin{array}{l}\text { Wilailak } \\
(2011)^{25} \\
\text { Healthy } \\
\text { controls }\end{array}$ & $\begin{array}{l}\text { General } \\
\text { U.S Adult } \\
\text { population } \\
\text { norms }\end{array}$ \\
\hline $\begin{array}{l}\text { Physical Well } \\
\text { Being }\end{array}$ & $24 \cdot 19(3 \cdot 90)$ & $26 \cdot 3(1 \cdot 84)$ & $20 \cdot 47(4 \cdot 66)$ & $22 \cdot 7(5 \cdot 4)$ \\
\hline $\begin{array}{l}\text { Social Family } \\
\text { Well Being }\end{array}$ & $19 \cdot 68(5 \cdot 28)$ & $24 \cdot 4(4 \cdot 76)$ & $17 \cdot 82(5 \cdot 62)$ & $19 \cdot 1(6 \cdot 8)$ \\
\hline $\begin{array}{l}\text { Emotional } \\
\text { Well Being }\end{array}$ & $19 \cdot 62(3 \cdot 54)$ & $21 \cdot 2(3 \cdot 11)$ & $17 \cdot 25(4 \cdot 35)$ & $19 \cdot 9(4 \cdot 8)$ \\
\hline $\begin{array}{l}\text { Function Well } \\
\text { Being }\end{array}$ & $21 \cdot 01(5 \cdot 15)$ & $26 \cdot 2(2 \cdot 53)$ & $19 \cdot 36(5 \cdot 81)$ & $18 \cdot 5(6 \cdot 8)$ \\
\hline $\begin{array}{l}\text { Overall FACT- } \\
\text { G }\end{array}$ & $82 \cdot 15(12 \cdot 86)$ & $98 \cdot 2(8 \cdot 98)$ & $72 \cdot 58(13 \cdot 80)$ & $80 \cdot 1(18 \cdot 1)$ \\
\hline
\end{tabular}

Within FACT-G scores there were no significant differences based on population characteristics; however patients who desired further fertility showed significantly lower FACT-G score in the emotional well-being domain $(p=0.02){ }^{26}$

Studies including patients either in remission or during active treatment are undermined by a lack of information on disease status. ${ }^{15,16,23}$ Two studies using the EORTC-QLQ-C30 and the SWLS demonstrate that chemotherapy significantly reduces overall HRQoL. ${ }^{15,23}$ The SWLS results show a lower overall score for chemotherapy patients versus non-chemotherapy patients $(21.7$ [95\% CI 13.9$29 \cdot 6]$ vs. $25 \cdot 0$ [95\% CI 18·2-31-9]). ${ }^{15}$

In a study of women with gynaecological cancer, $38 \%$ of whom had GTN, the overall HRQoL score using the EORTC QLQ-C30 was $48.5,{ }^{23}$ with the reference 
score for female cancer patients using EORTC QLQ C-30 being 59.3.29 Interestingly patients with metastasis reported significantly lower scores of HRQoL on the emotional $(p<0 \cdot 01)$, cognitive $(p<0 \cdot 01)$ and social functioning subscales, as well as significantly more severe scores of constipation $(p<0 \cdot 01)$ and financial impact items $(\mathrm{p}<0 \cdot 01){ }^{23}$

Utilising the WHO-QOL-BRE, Ferreira et al. included women needing chemotherapy (43\%)(78\% had single agent and 22\% multi agent). ${ }^{16}$ The overall HRQoL was good, and chemotherapy exerted no significant influence on HRQoL. However, patients receiving chemotherapy had a higher physical and psychological burden and needed more social support compared to the MP group. It is unclear when the patients completed the questionnaire, which may undermine the results.

\section{Physical Functioning}

Only one study includes a tool specific to the physical domain, the Menopausal Symptom Checklist (MSC). ${ }^{30}$ This study includes only 5 GTN patients out of a sample of 88, but menopausal symptoms are an extremely common side effect of multi-agent chemotherapy. ${ }^{31} 22-43 \%$ reported being very bothered by menopausal symptoms, most commonly vaginal dryness and hot flashes.

\section{Psychological Functioning}

Psychological HRQoL issues are the most extensively researched for GTN patients. ${ }^{15-18,20,22,27,30,32}$ Four studies used a sample of GTN patients in complete remission, $17,20,27,30$ while the others were a mixed sample between active treatment and complete remission. ${ }^{15,16,18,22,32}$ All studies included patients who had received either single or multi-agent treatment.

\section{GTN in complete remission}

The HADS, CES-D and IES-R validated tools showed no significant difference between MP and GTN, where across both groups $26 \%$ had elevated levels of anxiety (score $>8$ on HADS-A), and 22\% elevated levels of depression (score $>16$ on CES-D). ${ }^{17}$ Both these results exceed the 12-month local community rates of anxiety [17.9\%] and depression [7·1\%]. ${ }^{17}$ Anxiety levels were higher among women without a pregnancy subsequent to the GTD diagnosis $(p=0 \cdot 04) .{ }^{17}$ For women who have impaired fertility post any cancer treatment, the CES-D and IES show clinically significant levels of distress (76\%) and depression (26\%). ${ }^{30}$

\section{GTN - mixed sample of unknown disease status}

This group of studies again showed no statistically significant differences in psychological outcomes based on the need for chemotherapy. ${ }^{15,16,18,22}$ One study found a statistical difference $(\mathrm{p}<0.006)$ across several physical and emotional issues between those with metastatic and non-metastatic disease. ${ }^{21}$ 
General psychosocial outcomes and depression were assessed using the Mood Disturbance Scale and Psychosocial Response to Illness Scale and found 11\% of the MP group vs. $16 \%$ in the GTN group experienced depression. ${ }^{22}$ Although anxiety was more prominent than depression in both groups (MP 21\% and GTN $24 \%$ anxiety). ${ }^{21}$ Two studies used the BDI and both found significant levels of depression within the GTN subgroup. ${ }^{16,18}$

GTN patients with children had significantly better emotional functioning, 15, 22, 30 with significantly lower overall HADS scores attributable to a reduction in anxiety domains (HADS - A: children $=46.9 \%$, no children $=76 \% ; p=0 \cdot 04$ ). ${ }^{15}$ The CES-D found women with children to have less overall depression, with significantly lower CES-D scores $(\mathrm{P}<0 \cdot 047) .{ }^{30}$ In the psychosocial response to illness measure, women without children felt greater fear and a negative impact on self $(\mathrm{p}<0 \cdot 02){ }^{22}$

\section{$\underline{\text { Social Functioning }}$}

\section{Relationship Concerns and Sexual Function}

The majority of patients felt well supported and their relationship to be unchanged or closer following GTN. ${ }^{17,} 20,22,30,33$ However only one study used a validated measurement tool (Marital Satisfaction Survey - MSS). ${ }^{17}$ On a mixed sample of MP and GTN the MSS results were not significantly different across the two groups, showing overall satisfaction and support. However $26 \%$ reported a negative impact on their sex life. ${ }^{17}$

Women with GTN reported a significant level of sexual dysfunction. 15, 17, 20, 22, 27, 30,33 Three of the studies used validated tools and found sexual dysfunction compared to community controls using the Sexual History Form-12 (SHF-12), and the Female Sexual Functioning Index (FSFI) (Table VI). 15, 17, 30 Using the SHF12 , participants scored a mean of $0.52(95 \%$ CI $0 \cdot 35-0 \cdot 6)$ vs community control of $0 \cdot 49.15$

Table V: Summary of FSFI scores for GTN

\begin{tabular}{|l|l|l|}
\hline & $\begin{array}{l}\text { Carter et al. (2009) } \\
\text { GTN patients }- \text { only 6\% } \\
\text { of sample }\end{array}$ & $\begin{array}{l}\text { Stafford et al. }(2011)^{17} \\
\text { GTN n=27 }\end{array}$ \\
\hline FSFI mean (SD) & $19 \cdot 75(9 \cdot 83)$ & $20 \cdot 32(10 \cdot 45)$ \\
\hline
\end{tabular}

Lower score $>$ sexual dysfunction; $<26=$ dysfunction

Sexual dysfunction is common amongst GTN patients using non-validated study specific questionnaires, though without any comparison to normative data. ${ }^{20,22}$ The most extensive investigation of sexual dysfunction in GTN explored symptoms of sexual dysfunction according to treatment type and prior to GTN. ${ }^{33}$ Most women [94\%] were sexually active with 90\% satisfied with their sexual relations. However a majority reported at least one or more types of sexual dysfunction. The most common originated in the psychological state $170 \%$ absent or reduced desire, $65 \%$ frigidity), with less sexual disturbance of an organic or functional nature (52\% vaginismus, $42 \%$ dyspareunia), although $53 \%$ 
of patients reported these problems existed prior to diagnosis. 8\% of those receiving single agent chemotherapy reported transitory dysfunction, with the highest level of dysfunction observed in those treated with multi-agent chemotherapy and surgery. ${ }^{33}$

\section{$\underline{\text { Fertility Concerns }}$}

Fertility related distress appears to affect many women based on nonstandardised data; 38\% were worried about the next pregnancy $(88 \%$ feared another tumour), ${ }^{20} 50 \%$ fear becoming pregnant, ${ }^{22} 40 \%$ felt they had no control over their reproductive future, ${ }^{27}$ and $57 \%$ questioned their ability to have children. ${ }^{34}$

The Fertility Problem Inventory (FPI) in a small sample was unable to detect a clinically significant level of global stress. ${ }^{18}$ In a study focused on the impact of treatment-induced infertility, with a small sample of GTN cases (6\%), most women $(72 \%)$ reported clinically significant levels of distress related to their loss of fertility. ${ }^{30}$

\section{Synthesis of Qualitative Data}

The qualitative data was analysed to assess correlation with the quantitative data from the PROMs used in GTD in a process of data triangulation using the 'triangulation protocol' technique. ${ }^{35}$ As described by 0 'Caithan et al, a convergence coding framework consisting of HRQoL subheadings was used to display the findings emerging from each separate review. ${ }^{35}$

\section{$\underline{\text { Physical Functioning }}$}

Physical impact was most prevalent amongst GTN patients who underwent high dose chemotherapy, where a core theme was the reporting of problems persisting months and years after treatment. 36,37 These physical effects included peripheral neuropathy, hearing loss, breathing problems, fatigue affecting daily living, digestive problems and impaired concentration.

Participants reported a chemotherapy-induced negative impact on body image, resulting from scars from treatment, weight gain and change in body shape following chemotherapy. ${ }^{36,37}$ For those receiving single agent chemotherapy only, the majority found chemotherapy easier than expected. ${ }^{37}$ Amongst MP patients the data shows that women report physical complications, for example amenorrhea, are heightened by the impact of GTD. ${ }^{15}$

\section{Psychological Functioning}

The core themes were fear and anxiety, depression and self-esteem, with all four qualitative studies finding significant fear and anxiety relating to GTD. 15, 36-38 Fear experienced by MP and GTN patients is multi-faceted including recurrence of disease in a subsequent pregnancy, recurrence of the malignant tumour, fear 
of dying, fear of not being able to achieve a successful pregnancy, fear of chemotherapy and permanent damage resulting from this. ${ }^{15,36-38}$

Participants in all four qualitative studies expressed feelings associated with a depressed mood, including: 'sadness', 'upset', 'depressed', 'worthless', 'hopeless', 'grief'. Many experienced sleep disturbance as a symptom of on-going depression. ${ }^{37}$ Women cite the main reason for depression as the loss of a child through the molar pregnancy. ${ }^{15,37,38}$ Reasons for low mood are the diagnosis itself which caused uncertainty and confusion, along with the delay to future childbearing. ${ }^{15,37,38}$ For ultra high risk patients who required inpatient treatment over many weeks, the most cited cause for depression was separation from their children. ${ }^{36}$

Self-esteem was explored through questions about general outlook on life, and perceptions on their role as women. 33\% of women felt that the disease had had a profound negative effect on their roles as women. ${ }^{37}$ This negative effect was seen in women who reacted most strongly to their pregnancy failure. ${ }^{37,38}$ GTD impacted upon many women's general outlook on life, with many feeling more irritated and sad, with a more pessimistic outlook than before the disease. ${ }^{37,38}$ Women also reported a change in outlook for the better as a result of GTD, where some women found their experiences had helped them find new priorities in life and given them a sense of the their own strength and ability to cope. ${ }^{36,38}$

\section{Social Functioning}

Women found that one of the three most stressful aspects of GTN was the inability to communicate with family and friends due to the disease being unknown and uncommon. ${ }^{37}$ Participants felt they were 'met with disbelief when they mentioned cancer and chemotherapy. ${ }^{37}$ The supportive role of partners, family, friends and specialist GTD teams was seen as very important to those having high dose chemotherapy, especially around ensuring the children were cared for in their absence. ${ }^{36}$

The results around relationships are mixed, with an older study reporting minimal change in marital relationship (94\% unchanged, $4 \%$ improved). ${ }^{38}$ Partners who provided support were helpful and cited as a real source of strength. ${ }^{36}$ In a study from the Netherlands women reported they were left to cope with treatment on their own. ${ }^{37}$ In this study five couples separated due to the stress of coping with GTN, all of which were childless. ${ }^{37}$ In all studies MP and GTN had a negative impact on their sex life. ${ }^{15,36-38}$ The majority of patients reported a decline in sexual activity. ${ }^{36-38}$ The reasons behind this decline were expressed as a loss of interest or sexual desire partly due to a fear of pregnancy. 36

\section{Data Triangulation}

The core themes in the qualitative and quantitative data are well matched, but as would be expected, more detail emerges on the impact of GTD on HRQoL in the qualitative data. Within the overarching subheadings of physical, psychological and social functioning several new threads of data emerge. 
Table VI: Overview of themes emerging from qualitative data

\begin{tabular}{|c|c|c|}
\hline Physical functioning & $\begin{array}{l}\text { Psychological } \\
\text { Functioning }\end{array}$ & Social functioning \\
\hline $\begin{array}{ll}\text { - } & \text { Weight gain } \\
\text { - } & \text { Nerve damage } \\
\text { - } & \text { Hearing loss } \\
\text { - } & \text { Breathing problems } \\
\text { - } & \text { Fatigue that affected daily } \\
& \text { living } \\
\text { - } & \text { Digestive problems } \\
\text { - } & \text { Impaired concentration } \\
\text { - } & \text { Abnormal vaginal bleeding } \\
\text { - } & \text { Amenorrhoea } \\
\text { - } & \text { Body shape changes due to } \\
\text { - } & \text { Sleatment } \\
\text { - Sleep disorders }\end{array}$ & $\begin{array}{ll}\text { - } & \text { Grief } \\
\text { - } & \text { Fear of death } \\
\text { - } & \text { Resentment } \\
\text { - } & \text { Delay in childbearing }\end{array}$ & - Isolation \\
\hline
\end{tabular}

\section{Discussion}

Whilst long-term survivors of GTD generally have a favourable HRQoL, there is evidence suggesting the condition is a major source of physical, psychological and social morbidity. The impact of GTD on physical functioning is rarely explored, but the effect is clearly linked with increased disease burden and more intensive chemotherapy. Due to the use of a variety of instruments meta-analysis was not possible; however clinically significant levels of anxiety are consistently found across the MP and GTN groups. Anxiety is most commonly associated with fertility concerns, but also regular blood hCG monitoring, fear of malignancy, recurrence and death. Depression is reported less frequently and is related to grief over the loss of an unborn child, and also anger and resentment at the delay in childbearing. Having a child is linked with lower levels of anxiety and depression for GTD patients, though levels of anxiety and depression are the highest for those desiring further fertility and not having a child subsequent to GTD. For the majority of women their relationship appears unchanged or closer, but many report a degree of sexual dysfunction primarily due to lack of desire and loss of interest in sex, although this may have been present prior to the diagnosis.

The limitations of this analysis are that all of the studies are cross-sectional in design, providing only descriptive data at only one time-point with no longitudinal follow-up, and some studies use small heterogeneous samples. The patients include a mixture of GTD type, stage, treatment and time since diagnosis and utilise a range of different outcome tools . There are no studies explicitly investigating the short-term impact of GTD. Of the 19 studies evaluating GTN patients, 15 (79\%) only included patients in remission who have had single or multi-agent chemotherapy and/or surgery, and months or years following diagnosis. 
Of the tools used to measure HRQoL in GTD a significant proportion [22\%] are non-validated and the remainder are a mixture of generic and condition specific measures with varying degrees of validity and reliability. No disease-specific tool has been developed in GTD, instead a patchwork of different measures has been used to try and accommodate the unique and varied set of domains that are considered relevant to GTD patients. No single PROM can be recommended for use within GTD patients as a result of this review.

The scope of each instrument is reflected in the domains it covers, but it is difficult to assess the breadth and depth of their items, which vary from instrument to instrument and do not have equal weighting. As a result the use of these tools has several limitations in patients with GTD. For example, the EORTC QLQ-30 is broad in scope but does not address sexual function. The lack of any questions regarding fertility related distress, and the specific time frame of FACT-G (concerning the preceding seven days) does not allow the chronic, complex nature of the psychosocial sequelae of GTD to be expressed.

The process of triangulation between quantitative and qualitative data highlights the gaps in the data found by the PROMs used in studies where several themes had not been identified in the quantitative review. The triangulation process is limited due by the small number of qualitative studies in GTD, and also the fact that these studies offer limited scope to be representative of a wider GTD population, due to sample composition and methodology. The gaps identified by the qualitative review show that the content validity of the PROMs used in these studies is compromised for GTD patients.

\section{Conclusion}

This review has created a parallel synthesis of PROM data in GTD patients and provides insight into the impact of GTD on HRQoL. The results suggest the presence of significant physical, psychological and social sequelae associated with GTD, with clinically significant levels of anxiety, depression, sexual dysfunction and fertility concerns observed. Although, for long-term survivors of GTD HRQoL outcomes are at or above population norms. The overall body of evidence is plagued by heterogeneity within the included studies and undermined by bias introduced due to long time intervals since diagnosis and the cross-sectional rather than longitudinal nature of studies. Despite there only being a small number of qualitative studies, data triangulation produced several new HRQoL themes suggesting that the existing PROMs do not demonstrate content validity for the GTD population.

This review provides evidence of a need for a condition-specific PROM, enabling, monitoring an evaluating HRQoL issues associated with a GTD diagnosis in the context of supportive care provided by MDTs. As care pathways evolve, more evidence is needed on how to best allocate resources to support GTD patients during active monitoring, treatment and follow up based on prospective, longitudinal data, including the use of a condition-specific PROM that displays content and construct validity, reliability and responsiveness in the GTD population. 


\section{Contributors}

JI did the literature search and analysis, and led the writing of the systematic review. GJ and SCR contributed to the design and supervision of the review. BWH, MCW and JAT contributed their experience of clinical practice to ensure the relevance of findings, and the discussion of the implications for clinical practice. All authors edited the drafts and read, commented on, and approved the final review. JI had full access to all the data in the study and had final responsibility for the decision to submit for publication.

\section{Declaration of Interests}

We declare no competing interests.

\section{Acknowledgements}

We would like to acknowledge the contribution of Kam Singh, Annie Hills, and Sarah Gillett of the Sheffield Trophoblastic Centre whom assisted with the formation of ideas and editing of the final manuscript. This research was conducted during an MSc studentship with the National Institute of Health Research (NIHR) at the Sheffield School of Health and Related Research (SCHARR). 


\section{References}

1. Tidy, JA, \& Hancock, BW. The management of gestational trophoblastic neoplasia. Clinical guideline 38. Royal College of Obstetricians and Gynaecologists, London; 2010 Accessed from:

https://www.rcog.org.uk/globalassets/documents/guidelines/gt38management gestational0210.pdf in December 2012

2. Seckl MJ, Sebire NJ, Berkowitz RS. Gestational trophoblastic disease. The Lancet. 2010 Sep 3; 376(9742):717-29.

3. Ireson J, Singh K, Gillett S, Hills A, Everard J, Winter M, Coleman RE, Tidy J, Hancock BW. Evolution of a specialist gestational trophoblastic disease service with a major nursing component: the Sheffield, United Kingdom, experience. The Journal of Reproductive Medicine. 2013 Dec; 59(5-6): 195

4. US Food and Drug Administration. Guidance for industry: patient-reported outcome measures: use in medical product development to support labeling claims. Federal Registry. 2009 Dec; 74(235): 65132-3.

5. Black N. Patient reported outcome measures could help transform healthcare. British Medical Journal 2013; 346: f167.

6. Boyce MB, Browne JP, Greenhalgh J. The experiences of professionals with using information from patient-reported outcome measures to improve the quality of healthcare: a systematic review of qualitative research. British Medical Journal: quality \& safety. 2014 Feb 6:bmjqs-2013.

7. Fayers PM, Machin D. Quality of life: the assessment, analysis and reporting of patient-reported outcomes. John Wiley \& Sons; 2015 Nov 23.

8. Devlin NJ, Appleby J. Getting the most out of PROMS. Putting health outcomes at the heart of NHS decision-making. London: King's Fund. 2010.

9. Straatman J, Joosten PJ, Terwee CB, Cuesta MA, Jansma EP, Peet DL. Systematic review of patient-reported outcome measures in the surgical treatment of patients with esophageal cancer. Diseases of the Esophagus. 2015 Nov 1.

10. National Cancer Survivorship Initiative. Living with and beyond cancer: taking action to improve outcomes. London, UK: NCSI. 2013.

11. Department of Health Quality of Life of Cancer Survivors in England: Report on a pilot survey using Patient Reported Outcome Measures (PROMS). 2012 London: Department of Health

12. All Party Parliamentary Group on Cancer. Cancer across the Domains: Cancer priorities for the new NHS. 2013. Accessed from: 
http://www.macmillan.org.uk/documents/campaigns/canceracrossthedomains. pdf in July 2016.

13. Chen J, Ou L, Hollis SJ. A systematic review of the impact of routine collection of patient reported outcome measures on patients, providers and health organisations in an oncologic setting. BMC health services research. 2013 Jun 11; 13(1): 211.

14. Higgins JP, Green S, editors. Cochrane handbook for systematic reviews of interventions. John Wiley \& Sons; 2011 Aug 24. Available from www.handbook.cochrane.org.

15. Petersen RW, Ung K, Holland C, Quinlivan JA. The impact of molar pregnancy on psychological symptomatology, sexual function, and quality of life.

Gynecologic Oncology. 2005 May 31; 97(2): 535-42.

16. Ferreira EG, Maestá I, Michelin OC, de Paula, RC, Consonni M, and Rudge, MV. Assessment of quality of life and psychologic aspects in patients with gestational trophoblastic disease. The Journal of Reproductive Medicine 2009. Oct; 54(4): 239-244.

17. Stafford L, McNally OM, Gibson P, Judd F. Long-term psychological morbidity, sexual functioning, and relationship outcomes in women with gestational trophoblastic disease. International Journal of Gynecological Cancer. 2011 Oct 1; 21(7): 1256-63.

18. Di Mattei VE, Carnelli L, Bernardi M, Bagliacca EP, Zucchi P, Lavezzari L, Giorgione V, Ambrosi A, Mangili G, Candiani M, Sarno L. An investigative study into psychological and fertility sequelae of gestational trophoblastic disease: the impact on patients' perceived fertility, anxiety and depression. PloS one. 2015 Jun 1; 10(6): e0128354.

19. Bjelland I, Dahl AA, Haug TT, Neckelmann D. The validity of the Hospital Anxiety and Depression Scale: an updated literature review. Journal of Psychosomatic Research. 2002 Feb 28; 52(2): 69-77.

20. Berkowitz RS, Marean AR, Hamilton N, Bernstein M, Engle Jr RP, Sandman G, Goldstein DP. Psychological and Social Impact of Gestational Trophoblastic Neoplasia. The Journal of Reproductive Medicine, 1980. July; 25(1): 14-16.

21. Lok CA, Donker M, Calff MM, Massuger LF, Ansink AC. Psychologic impact of follow-up after low-risk gestational trophoblastic disease. The Journal of Reproductive Medicine. 2010 Dec; 56(1-2): 47-52.

22. Wenzel L, Berkowitz R, Robinson S, Bernstein M, Goldstein, D. The psychological, social, and sexual consequences of gestational trophoblastic disease. Gynecologic Oncology, 1992. 46(1): 74-81. 
23. Zhao H, Kanda K, Liu SJ, Mao XY. Evaluation of quality of life in Chinese patients with gynaecological cancer: assessments by patients and nurses. International journal of nursing practice. $2003 \mathrm{Feb} 1 ; 9(1)$ : 40-8.

24. Cagayan MS, Llarena RT. Quality of life of gestational trophoblastic neoplasia survivors: a study of patients at the Philippine General Hospital trophoblastic disease section. The Journal of Reproductive Medicine. 2009 Dec; 55(7-8): 321-6.

25. Wilailak S, Lertkhachonsuk AA, Lohacharoenvanich N, Luengsukcharoen SC, Jirajaras M, Likitanasombat P, Sirilerttrakul S. Quality of life in gynecologic cancer survivors compared to healthy check-up women. Journal of Gynecologic Oncology. 2011 Jun 30; 22(2): 103-9.

26. Leenharattanarak P, Lertkhachonsuk R. Quality of life in gestational trophoblastic neoplasia patients after treatment in Thailand. Asian Pacific Journal of Cancer Prevention. 2014; 15(24): 10871-4.

27. Wenzel L, Berkowitz RS, Newlands E, Hancock B, Goldstein DP, Seckl MJ, Habbal R, Bernstein M, Kluhsman B, Kulchak-Rahm A, Strickland S. Quality of life after gestational trophoblastic disease. The Journal of Reproductive Medicine, 2002. 47(5), pp.387-394.

28. Brucker PS, Yost K, Cashy J, Webster K, Cella D. General population and cancer patient norms for the Functional Assessment of Cancer Therapy-General (FACT-G). Evaluation \& the Health Professions. 2005 Jun; 28(2): 192-211.

29. Scott NW, Fayers PM, Aaronson NK, Bottomley A, de Graeff A, Groenvold M, Gundy C, Koller M, Petersen MA, Sprangers MA. EORTC QLQ-C30 reference values.

30. Carter J, Chi DS, Brown CL, Abu-Rustum NR, Sonoda Y, Aghajanian C, Levine DA, Baser RE, Raviv L, Barakat RR. Cancer-related infertility in survivorship. International Journal of Gynecological Cancer. 2010 Jan 1; 20(1): 2-8.

31. Seckl MJ, Rustin GJ. Late toxicity after therapy of gestational trophoblastic tumours. Gestational Trophoblastic Disease. 3rd ed. London: International Society for the Study of Trophoblastic Disease. 2003:470-84.

32. Hengrasmee P, Padungsutt P, Boriboonhirunsarn D. Depression among gynecologic cancer patients at Siriraj Hospital: prevalence and associated factors. Journal of the Medical Association of Thailand. 2004 Oct; 87:S74-9.

33. Cagayan MS. Sexual dysfunction as a complication of treatment of gestational trophoblastic neoplasia. The Journal of Reproductive Medicine. 2008 Aug; 53(8): 595-9.

34. Stafford L, Judd F. What do women with gestational trophoblastic disease understand about the condition? International Journal of Gynecological Cancer. 2011 Jan 1; 21(1): 161-6. 
35 O'Cathain A, Murphy E, Nicholl J. Three techniques for integrating data in mixed methods studies. British Medical Journal. 2010 Sep 17; 341: c4587.

36. Singh K, Warnock C, Ireson J, Strickland S, Short D, Seckl M, Hancock BW. The experience of women with gestational trophoblastic neoplasia treated with high dose chemotherapy and stem cell rescue; a qualitative study. Oncology Nursing Forum, 2017. May 44(3): 375-383.

37. Flam F, Magnusson C, Lindstedt VL, Von Schoultz B. Psychosocial impact of persistent trophoblastic disease. Journal of Psychosomatic Obstetrics \& Gynecology, 1993. 14(4): 241-248.

38. Ngan HY, Tang GW. Psychosocial aspects of gestational trophoblastic disease in Chinese residents of Hong Kong. The Journal of Reproductive Medicine. 1986 Mar; 31(3): 173-8.

\section{Useful websites for GTD:}

Sheffield Trophoblastic Centre website:

http://stdc.group.shef.ac.uk/

Charing Cross Trophoblastic Centre website:

http://www.hmole-chorio.org.uk/ 
